\title{
THE AUSLANDER-REITEN QUIVER OF A SIMPLE CURVE SINGULARITY
}

BY

\author{
ERNST DIETERICH AND ALFRED WIEDEMANN
}

\begin{abstract}
With any simple curve singularity (plane, complex, affine-algebraic) of Dynkin-type $\Delta$ we associate the category of all finitely generated torsionfree modules over its complete local ring. For each of these module categories we calculate the Auslander-Reiten quiver. We suggest the construction of the "twisted quiver" of a quiver with involution and valuation of arrows which gives rise to a (purely combinatorial) one-to-one correspondence between the Auslander-Reiten quiver and the Dynkin diagram $\Delta$.
\end{abstract}

Let $k$ be an algebraically closed field of characteristic 0 and $\Delta$ a Dynkin diagram. The simple plane curve singularities over $k$ are given by the following polynomials $f_{\Delta}(X, Y)[\mathbf{A r} 74]:$

$$
\begin{array}{lll}
\mathbf{A}_{n}: & X^{n+1}+Y^{2} & (n=1,2,3, \ldots), \\
\mathbf{D}_{n}: & X^{n-1}+X Y^{2} & (n=4,5,6, \ldots), \\
\mathbf{E}_{6}: & X^{4}+Y^{3}, \\
\mathbf{E}_{7}: & X^{3} Y+Y^{3}, \\
\mathbf{E}_{8}: & X^{5}+Y^{3} .
\end{array}
$$

With each singularity of type $\Delta$ we associate its complete local ring $\Lambda_{\Delta}=$ $k[[X, Y]] /\left(f_{\Delta}(X, Y)\right)$ and the category $\mathscr{S}_{\Lambda_{\Delta}}$ of all finitely generated $S$-torsionfree $\Lambda_{\Delta}$-modules, $S$ being the set of all non-zero-divisors of $\Lambda_{\Delta}$. This paper's aim is to investigate the Auslander-Reiten structure of $\mathscr{S}_{\Lambda_{\Delta}}$ : We shall see that the AuslanderReiten quiver of $\mathscr{S}_{\Lambda_{\Delta}}$ corresponds to the extended Dynkin diagram $\tilde{\Delta}$, whereas its stable subquiver corresponds to $\Delta$. This correspondence is effected by way of a purely combinatorial construction which assigns to a quiver with involution and valuation of arrows its twisted quiver.

The authors were motivated towards this investigation by the recent observation of Greuel and Knörrer that $\mathscr{S}_{\Lambda_{\Delta}}$ has only a finite number of isomorphism classes of indecomposable objects [ $\mathbf{G r} / \mathbf{K n 8 5}$ ]. Independently and by different methods they both calculated the Auslander-Reiten quiver of $\mathscr{S}_{\Lambda_{\Delta}}$ for all Dynkin diagrams $\Delta$. The present paper is based upon the first author's manuscript. He observed the connection between the Auslander-Reiten quiver of $\mathscr{S}_{\Lambda_{\Delta}}$ and the Dynkin diagram $\Delta$ (see Theorems A and B), whereas the second author focused upon a characterization of

Received by the editors September 12, 1984. The content of this paper has been presented at the workshop on representation theory of finite groups and finite-dimensional algebras, supported by the Deutsche Forschungsgemeinschaft, at Essen University on June 22, 1984.

1980 Mathematics Subject Classification. Primary 13C05; Secondary 14H20, $14 \mathrm{H} 45$. 
the complete local rings $\Lambda_{\Delta}$ among the wider class of all local representation-finite Gorenstein orders [Wi84]. We are indebted to H. Knörrer for disseminating the second author's results, thus leading to the present joint paper.

1. Statement of the result. Before we can present the result we have to collect a few notions from the language of quivers.

Recall that a quiver $Q=\left(Q_{0}, Q_{1}\right)$ is given by a set of "points" $Q_{0}$ and a set of "arrows" $Q_{1}$, linked by a map $Q_{1} \rightarrow Q_{0} \times Q_{0}$ which assigns to each arrow its "starting point" and its "end point". We write $x \stackrel{\alpha}{\rightarrow} y$ in order to indicate that the arrow $\alpha$ has starting point $x$ and end point $y$.

In our context, a translation quiver $Q=(Q, \tau)$ is a quiver $Q$ together with two distinguished subsets $Q_{0}^{\prime}, Q_{0}^{\prime \prime}$ of $Q_{0}$ and a bijective map $\tau: Q_{0}^{\prime} \rightarrow Q_{0}^{\prime \prime}$, satisfying the following conditions:

(a) $Q$ contains no multiple arrows (i.e. the map $Q_{1} \rightarrow Q_{0} \times Q_{0}$ is injective).

(b) For all $x \in Q_{0}^{\prime}$, the set of predecessors of $x$ equals the set of successors of $\tau x$.

(Observe that this notion is slightly more general than the notion of translation quiver introduced into representation theory by Riedtmann [Rie80] and Bongartz and Gabriel [Bo/Ga82], because in the above definition we do not exclude "loops" (i.e. arrows for which starting point and end point coincide).) Given a translation quiver $Q=(Q, \tau)$, we call $\tau$ its "translation map", the points in $Q_{0} \backslash Q_{0}^{\prime}$ its "projective points" and the points in $Q_{0} \backslash Q_{0}^{\prime \prime}$ its "injective points". Moreover, a point $x$ is said to be "stable" if $\tau^{n} x$ is defined for all $n \in \mathbf{Z}$.

With any translation quiver $Q$ we associate its stable subquiver $Q_{s}$ which is the full subquiver of $Q$ consisting of all stable points of $Q$. Note that the restriction of $\tau$ gives rise to an automorphism of the stable subquiver.

An involution quiver $Q=(Q, \gamma)$ is a quiver $Q$ together with a full subquiver $Q_{s}$ and an involution $\gamma$ of $Q_{s}$. We call $Q_{s}$ the stable subquiver of the involution quiver $Q$.

The subject matter of the present paper is the Auslander-Reiten quiver of $\Lambda_{\Delta}$, denoted by $\mathscr{A}\left(\Lambda_{\Delta}\right)$. It is defined as follows: The set of points of $\mathscr{A}\left(\Lambda_{\Delta}\right)$ is given by the set of all isomorphism-classes [ $M$ ] of indecomposable objects $M$ in $\mathscr{S}_{\Lambda_{\Delta}}$. For any two points $[M],\left[M^{\prime}\right]$ there exists an arrow $[M] \rightarrow\left[M^{\prime}\right]$ in $\mathscr{A}\left(\Lambda_{\Delta}\right)$ if and only if there exists an irreducible map in $\operatorname{Hom}_{\Lambda_{\Delta}}\left(M, M^{\prime}\right)$. (Recall that a homomorphism $\varphi: \quad M \rightarrow M^{\prime}$ between indecomposable modules $M, M^{\prime}$ is said to be "irreducible" if it is not an isomorphism and if it admits no nontrivial factorization; that is to say, whenever $\varphi=\eta \zeta, \zeta: M \rightarrow X, \eta: X \rightarrow M^{\prime}, X \in \mathscr{S}_{\Lambda_{\Delta}}$, then either $\zeta$ is a splittable monomorphism or $\eta$ is a splittable epimorphism.) In $\S 2$ we shall prove that $\Lambda_{\Delta}$ can be viewed as an $R$-order, where $R$ is a complete discrete valuation ring which is a subring of $\Lambda_{\Delta}$, such that $\mathscr{S}_{\Lambda_{\Delta}}$ equals the category of $\Lambda_{\Delta}$-lattices. Consequently, $\mathscr{S}_{\Lambda_{\Delta}}$ has Auslander-Reiten sequences and $\mathscr{A}\left(\Lambda_{\Delta}\right)$ is a translation quiver. We denote its stable subquiver by $\mathscr{A}_{s}\left(\Lambda_{\Delta}\right)$.

Aiming at a description of the Auslander-Reiten quiver of $\Lambda_{\Delta}$ we have to introduce a construction which, starting from a quiver endowed with additional combinatorial data, yields a new quiver. Let $Q$ be a quiver, $\gamma$ an involution of $Q$ and $\delta: Q_{1} \rightarrow\{0,1\}$ a valuation of the arrows, subject to the condition that, for all 
$\alpha \in Q_{1}, \delta(\alpha)=1$ only if $\gamma(\alpha)=\alpha$. Hence, we are given an operation of the cyclic group in two elements $G=\langle g\rangle$ on $Q$, by way of $g \mapsto \gamma$. For every $x \in Q_{0} \cup Q_{1}$ we denote by $G x$ the $G$-orbit of $x$ and by $G_{x}$ the stabilizer of $x$ under this $G$-operation. Then the twisted quiver $Q[\gamma, \delta]$ of $(Q, \gamma, \delta)$ is defined as follows:

$$
\begin{gathered}
Q[\gamma, \delta]_{0}:=\left\{(G x, s) \mid x \in Q_{0}, s \in G_{x}\right\}, \\
Q[\gamma, \delta]_{1}:=\left\{(G \alpha, s, t):(G x, s) \rightarrow(G y, t) \mid \alpha \in Q_{1}, \alpha: x \rightarrow y,\right. \\
\left.s=g^{i}, t=g^{j}, j-i \equiv \delta(\alpha) \quad\left(\bmod \left|G_{x} \cap G_{y}\right|\right)\right\} .
\end{gathered}
$$

It is easily verified that $Q[\gamma, \delta]$ is well defined. Observe that with the twisted quiver $Q[\gamma, \delta]$ an involution $\gamma^{\prime}$ of $Q[\gamma, \delta]$ is always given in a natural way. Namely, $\gamma^{\prime}(G x, s)=\left(G x, s^{\prime}\right)$ and $\gamma^{\prime}(G \alpha, s, t)=\left(G \alpha, s^{\prime}, t^{\prime}\right)$, subject to $\left\{s, s^{\prime}\right\}=G_{x}$ and $\left\{t, t^{\prime}\right\}=G_{y}$. Moreover, it is easy to see that for each twisted quiver $Q[\gamma, \delta]$ there exists a valuation of arrows $\delta^{\prime}: Q[\gamma, \delta]_{1} \rightarrow\{0,1\}$ such that $(Q[\gamma, \delta])\left[\gamma^{\prime}, \delta^{\prime}\right] \cong Q$. However, in general $\delta^{\prime}$ is not unique.

In a situation which is closely related to the above we shall also need the device of the twisted quiver. Let $(Q, \gamma, \delta)$ be given as above and let $n \in Q_{0}$. The one-point extension of $Q$ (with respect to $n$ and $\gamma$ ), denoted by $Q(n)$, is the quiver defined by $Q(n)_{0}:=Q_{0} \cup\{*\}$ and $Q(n)_{1}:=Q_{1} \cup\{n \rightarrow *, * \rightarrow \gamma(n)\}$. Moreover, the twisted quiver of a one-point extension is by definition the one-point extension of the twisted quiver. To be precise, $Q(n)[\gamma, \delta]:=Q[\gamma, \delta](G n, 1)$.

After these generalities let us now focus upon a distinguished set of quivers, each of them endowed with an involution, a valuation of arrows and a one-point extension. Namely, let $\Delta$ be a Dynkin diagram, embedded in its extended Dynkin diagram $\tilde{\Delta}$. Choose a point $n_{\Delta}$ in $\Delta$ which, in $\tilde{\Delta}$, is neighbour to the extending point of $\tilde{\Delta}$. Then we define:

$$
\begin{aligned}
& Q_{\Delta}:=\mathbf{Z} \Delta / \mathbf{Z} \quad(\text { see }[\mathbf{R i e 8 0 a}] \text { or Figure } 1 \text { for definition }), \\
& \gamma_{\Delta}:= \begin{cases}\text { reflection, } & \text { if } \Delta=\mathbf{A}_{n}, \mathbf{D}_{2 m+1}, \mathbf{E}_{6}, \\
\text { identity, } & \text { if } \Delta=\mathbf{D}_{2 m}, \mathbf{E}_{7}, \mathbf{E}_{8},\end{cases} \\
& \delta_{\Delta}(\alpha):= \begin{cases}1, & \text { if } \gamma_{\Delta}(\alpha)=\alpha \text { and } \alpha \text { points towards the branching point of } \Delta, \\
0, & \text { otherwise, }\end{cases} \\
& Q_{\Delta}:=Q_{\Delta}\left(n_{\Delta}\right) .
\end{aligned}
$$

Observe that the isomorphism type of $Q_{\tilde{\Delta}}$ is independent of the choice of $n_{\Delta}$. The quivers $Q_{\Delta} \subset Q_{\bar{\Delta}}$ and the axes for the reflections $\gamma_{\Delta}$ are shown in Figure 1. Moreover, we indicate the valuations $\delta_{\Delta}$ by marking with " 1 " those arrows $\alpha \in\left(Q_{\Delta}\right)_{1}$ for which $\delta_{\Delta}(\alpha)=1$.

Observe that $\left(Q_{\tilde{\Delta}}, \gamma_{\Delta}\right)$ is an involution quiver with stable subquiver $\left(Q_{\Delta}, \gamma_{\Delta}\right)$, whereas $\left(Q_{\Delta}\left[\gamma_{\Delta}, \delta_{\Delta}\right], \gamma_{\Delta}^{\prime}\right)$ is a translation quiver with stable subquiver $\left(Q_{\Delta}\left[\gamma_{\Delta}, \delta_{\Delta}\right], \gamma_{\Delta}^{\prime}\right)$ for each Dynkin diagram $\Delta$.

We are now in the position to formulate the result of the present paper. 
458

ERNST DIETERICH AND ALFRED WIEDEMANN

$\mathbf{A}_{2 m}$ :

$\mathbf{A}_{2 m+1}$ :

$\mathbf{D}_{2 m}$ :

$\mathbf{D}_{2 m+1}$ :

$\mathbf{E}_{6}$ :

$\mathbf{E}_{7}$ :

$\mathbf{E}_{8}$ :
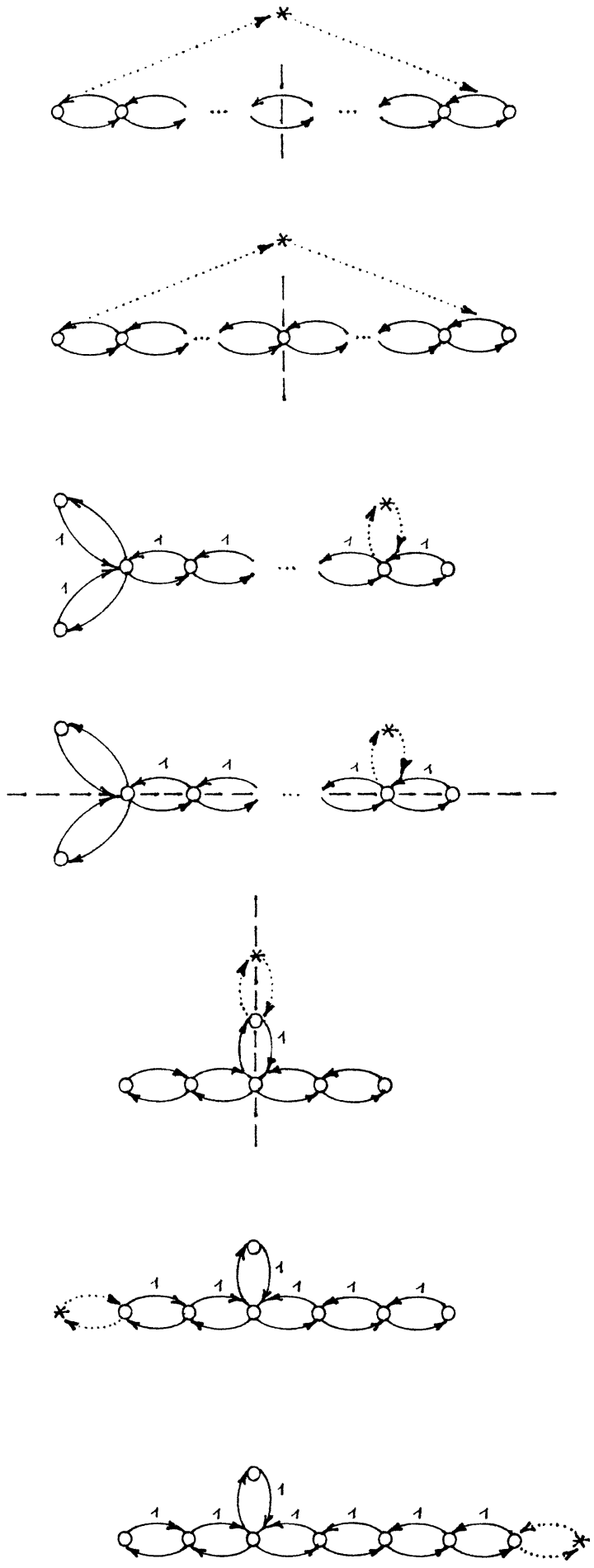

Figure 1 
Theorem A. Let $\Delta$ be a Dynkin diagram, $\Delta \neq \mathbf{A}_{1}$. Then the following translation quivers are isomorphic:

(i) $\mathscr{A}\left(\Lambda_{\Delta}\right) \cong Q_{\Delta}\left[\gamma_{\Delta}, \delta_{\Delta}\right]$,

(ii) $\mathscr{A}_{s}\left(\Lambda_{\Delta}\right) \cong Q_{\Delta}\left[\gamma_{\Delta}, \delta_{\Delta}\right]$.

Thus Theorem A gives a complete and explicit description of the AuslanderReiten quiver of $\Lambda_{\Delta}$ in a purely combinatorial way, starting from $\Delta$, by way of the twisted quiver of $\mathbf{Z} \Delta / \mathbf{Z}$. It implies in particular that $\mathscr{A}\left(\Lambda_{\Delta}\right)$ is a one-point extension of $\mathscr{A}_{s}\left(\Lambda_{\Delta}\right)$ and that the Auslander-Reiten translation $\tau$ is an involution of $\mathscr{A}_{s}\left(\Lambda_{\Delta}\right)$. Now define a valuation of arrows $\delta: \mathscr{A}_{s}\left(\Lambda_{\Delta}\right) \rightarrow\{0,1\}$ by

$$
\delta(\alpha):= \begin{cases}1 & \text { if } \alpha \text { is a loop } \\ 0 & \text { otherwise }\end{cases}
$$

Then we have

Theorem B. Let $\Delta$ be a Dynkin diagram, $\Delta \neq \mathbf{A}_{1}$. Then the following involution quivers are isomorphic:

(i) $\mathscr{A}\left(\Lambda_{\Delta}\right)[\tau, \delta] \cong Q_{\tilde{\Delta}}$,

(ii) $\mathscr{A}_{s}\left(\Lambda_{\Delta}\right)[\tau, \delta] \cong Q_{\Delta}$.

Thus, for any simple plane curve singularity of type $\Delta, \Delta \neq \mathbf{A}_{1}$, Theorem $\mathrm{B}$ shows that the complete local ring of the singularity uniquely determines the Dynkin diagram $\Delta$ by way of the twisted quiver of its Auslander-Reiten quiver.

In case $\Delta=\mathbf{A}_{1}$, the second statement of both theorems is still valid. However, in order to obtain $\mathscr{A}\left(\Lambda_{\mathbf{A}_{1}}\right)$, one has to add two more arrows to $Q_{\tilde{\mathbf{A}}_{1}}\left[\gamma_{\mathbf{A}_{1}}, \delta_{\mathbf{A}_{1}}\right]$ :

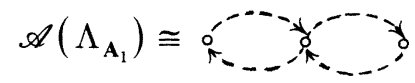

In both theorems the second statement is an immediate consequence of the first, because isomorphic translation quivers and isomorphic involution quivers have isomorphic stable subquivers. Moreover, Theorem B follows easily from Theorem A: One simply has to verify that $\left(Q_{\tilde{\Delta}}\left[\gamma_{\Delta}, \delta_{\Delta}\right]\right)\left[\gamma_{\Delta}^{\prime}, \delta\right] \cong Q_{\tilde{\Delta}}$, working through the twisting construction twice, for all Dynkin diagrams $\Delta$.

Hence the core of the above results is Theorem A(i). A proof for this statement will be given in the remaining part of the present paper, proceeding along the following line: In $\$ 2$ we shall verify that each complete local ring $\Lambda_{\Delta}$ is a Gorenstein-order (with respect to a suitably chosen subring $R$ ) and that $\mathscr{S}_{\Lambda_{\Delta}}$ coincides with the category of $\Lambda_{\Delta}$-lattices. Consequently, any tool available in the theory of lattices over orders can be applied to the category $\mathscr{S}_{\Lambda_{\Delta}}$. In this respect we shall heavily draw from two sources, namely the theory of Auslander-Reiten sequences and the reduction functor due to Green-Reiner and Ringel-Roggenkamp [Gr/Re78, Rin/Ro79]. We shall combine these concepts by observing that the dimension-mapping (which will be defined by use of the reduction functor) is additive on Auslander-Reiten sequences. In $\$ 3$ we shall explicitly construct the Auslander-Reiten quiver of $\Lambda_{\Delta}$ on this basis, using the simple combinatorics of the 
dimension-mapping as far as possible, aided by direct calculations (in either of the categories which are related under the reduction functor) if necessary. For each Dynkin diagram $\Delta$, the isomorphism of translation quivers asserted in Theorem A(i) will then be evident.

Two remarks relating the construction of the twisted quiver to other parts of representation theory are in order.

1 . Let $Q$ be a quiver such that its underlying graph is a Dynkin diagram $\Delta$ and let $k Q$ be the path-algebra of $Q$. Then the Auslander-Reiten quiver of $k Q$ is a full subquiver of $\mathbf{Z} \Delta$ which admits a purely combinatorial description involving the Coxeter number $c_{\Delta}$ of $\Delta$ and an involution $\sigma_{\Delta}$ of $\Delta[\mathbf{G a 8 0 , ~ R i n 8 0 ] . ~ I t ~ t u r n s ~ o u t ~ t h a t ~ t h e ~}$ involutions $\sigma_{\Delta}$ and $\gamma_{\Delta}$ (as defined above) coincide. This has an explanation in the second author's necessary conditions for configurations of Auslander-Reiten quivers of local Gorenstein orders using the same additive functions on $\mathbf{Z} \Delta$ as one does in the computation of the Auslander-Reiten quiver of $k Q$ [Wi84].

2. Let $(Q, \gamma, \delta)$ be a quiver with involution and valuation of arrows. Let $k Q$ be its path-algebra and denote by $\gamma \delta$ the involution of $k Q$ which is given by $\gamma \delta(x)=\gamma(x)$, $\gamma \delta(\alpha)=(-1)^{\delta(\alpha)} \gamma(\alpha)$ for all $x \in Q_{0}, \alpha \in Q_{1}$. Then the twisted group algebra $(k Q)[\gamma \delta]$ of $k Q$ (with respect to $\gamma \delta$ ) is Morita-equivalent to the path-algebra $k(Q[\gamma, \delta])$ of the twisted quiver of $Q$ (with respect to $(\gamma, \delta))$ (see [Re/Ri85]).

2. Proof of Theorem A (first part): $\mathscr{S}_{\Lambda}$ is a lattice-category. Let us fix some notation. With each Dynkin diagram $\Delta$ we associate the following data:

$C=C_{\Delta}=$ plane singular curve with equation $f_{\Delta}(X, Y)=0$.

$r=r_{\Delta}=$ number of irreducible components of $C_{\Delta}$.

$\Lambda=\Lambda_{\Delta}=k[[X, Y]] /\left(f_{\Delta}(X, Y)\right)=$ complete local ring of the singularity $\left(C_{\Delta}, 0\right)$.

$\Gamma=\Gamma_{\Delta}=\prod_{i=1}^{r_{\Delta}} k[[T]]=\prod_{i=1}^{r_{\Delta}} \Gamma e_{i}$.

$A=A_{\Delta}=\prod_{i=1}^{r_{\Delta}} k((T))=\prod_{i=1}^{r_{\Delta}} A e_{i}=$ total quotient ring of $\Gamma$.

Throughout the remaining part of the present paper, whenever a statement is valid for all Dynkin diagrams $\Delta$, we shall omit the subscript $\Delta$ in order to simplify notation.

LEMMA 2.1. For each Dynkin diagram $\Delta$ there is a ring monomorphism $\iota: \Lambda \hookrightarrow \Gamma$ which maps $\Lambda$ onto the following subring $\iota \Lambda$ of $\Gamma$ :

$$
\begin{array}{ll}
\mathbf{A}_{2 m}: & k\left[\left[T^{2}, T^{2 m+1}\right]\right] \subset k[[T]], \\
\mathbf{A}_{2 m-1}: & k\left[\left[(T, T),\left(T^{m},-T^{m}\right)\right]\right] \subset k[[T]] \times k[[T]], \\
\mathbf{D}_{2 m}: & k\left[\left[\left(i T,-T^{m-1}, T^{m-1}\right),(0, T, T)\right]\right] \subset k[[T]] \times k[[T]] \times k[[T]], \\
\mathbf{D}_{2 m+1}: & k\left[\left[\left(T, T^{2 m-1}\right),\left(0, T^{2}\right)\right]\right] \subset k[[T]] \times k[[T]], \\
\mathbf{E}_{6}: & k\left[\left[T^{3}, T^{4}\right]\right] \subset k[[T]], \\
\mathbf{E}_{7}: & k\left[\left[\left(T, T^{2}\right),\left(0, T^{3}\right)\right]\right] \subset k[[T]] \times k[[T]], \\
\mathbf{E}_{8}: & k\left[\left[T^{3}, T^{5}\right]\right] \subset k[[T]] .
\end{array}
$$


Proof. For each Dynkin diagram $\Delta$ we parametrize each irreducible component $C_{i}$ of the curve $C$ individually:

$$
\begin{array}{ll}
\mathbf{A}_{2 m}: & \varphi_{1}: k \rightarrow C_{1}, \quad \varphi_{1}(t)=\left(-t^{2}, t^{2 m+1}\right), \\
\mathbf{A}_{2 m-1}: & \varphi_{1}: k \rightarrow C_{1}, \quad \varphi_{1}(t)=\left(t, i t^{m}\right), \\
& \varphi_{2}: k \rightarrow C_{2}, \quad \varphi_{2}(t)=\left(t,-i t^{m}\right), \\
\mathbf{D}_{2 m}: \quad & \varphi_{1}: k \rightarrow C_{1}, \quad \varphi_{1}(t)=(0, t), \\
& \varphi_{2}: k \rightarrow C_{2}, \quad \varphi_{2}(t)=\left(t, i t^{m-1}\right), \\
& \varphi_{3}: k \rightarrow C_{3}, \quad \varphi_{3}(t)=\left(t,-i t^{m-1}\right), \\
\mathbf{D}_{2 m+1}: \quad \varphi_{1}: k \rightarrow C_{1}, \quad \varphi_{1}(t)=(0, t), \\
& \varphi_{2}: k \rightarrow C_{2}, \quad \varphi_{2}(t)=\left(-t^{2}, t^{2 m-1}\right), \\
\mathbf{E}_{6}: & \varphi_{1}: k \rightarrow C_{1}, \quad \varphi_{1}(t)=\left(t^{3},-t^{4}\right), \\
\mathbf{E}_{7}: & \varphi_{1}: k \rightarrow C_{1}, \quad \varphi_{1}(t)=(t, 0), \\
& \varphi_{2}: k \rightarrow C_{2}, \quad \varphi_{2}(t)=\left(-t^{2}, t^{3}\right), \\
\mathbf{E}_{8}: & \varphi_{1}: k \rightarrow C_{1}, \quad \varphi_{1}(t)=\left(t^{3},-t^{5}\right) .
\end{array}
$$

This parametrization gives rise to a ring monomorphism

$$
\kappa: k[X, Y] /\left(f_{\Delta}(X, Y)\right) \hookrightarrow \prod_{i=1}^{r_{\Delta}} k[T]_{i},
$$

which extends to the asserted ring monomorphism

$$
\iota: k[[X, Y]] /\left(f_{\Delta}(X, Y)\right) \hookrightarrow \prod_{i=1}^{r_{\Delta}} k[[T]]_{i} .
$$

It is easily verified that $\iota \Lambda$ is the subring of $\Gamma$ specified in the lemma. Q.E.D.

Henceforth we shall identify $\Lambda$ with $\iota \Lambda$. With $\Lambda$ we shall always associate the embeddings $\Lambda \subset \Gamma \subset A$.

For each Dynkin diagram $\Delta$ denote the first generating element of $\Lambda$ (in the sense of Lemma 2.1) by $\pi=\pi_{\Delta}: \pi_{\mathbf{A}_{2 m}}=T^{2}, \pi_{\mathbf{A}_{2 m-1}}=(T, T), \pi_{\mathbf{D}_{2 m}}=\left(i T,-T^{m-1}, T^{m-1}\right)$, $\pi_{\mathbf{D}_{2 m+1}}=\left(T, T^{2 m-1}\right), \pi_{\mathbf{E}_{6}}=T^{3}, \pi_{\mathbf{E}_{7}}=\left(T, T^{2}\right), \pi_{\mathbf{E}_{8}}=T^{3}$. Fix the subring $R=k[[\pi]]$ of $\Lambda$ and let $K$ be its quotient field. Then we have

LEMMA 2.2. (i) $A$ is a finite-dimensional separable $K$-algebra.

(ii) $\Lambda$ is an $R$-order in $A$ and $\Gamma$ is the maximal order in $A$.

(iii) The category $\mathscr{S}_{\Lambda}$ coincides with the category of $\Lambda$-lattices.

Proof. Denote by $S_{\Gamma}$ the set of all non-zero-divisors of $\Gamma$. By choice of $R$, the following statements are true for each Dynkin diagram $\Delta$ :

(1) $R$ is a complete discrete valuation ring.

(2) $\Gamma$ is finitely generated over $R$.

(3) $K \Gamma=A$. 
(4) There exists a natural number $l$ such that $\pi^{l} \Gamma \subset \Lambda$.

(5) $R \backslash\{0\} \subset S_{\Gamma}$.

Assertions (i) and (ii) are easy consequences of (1)-(4). To prove (iii), observe that $S=S_{\Gamma} \cap \Lambda$ (in view of (4)), hence $R \backslash\{0\} \subset S$ (in view of (5)). Therefore each finitely generated $S$-torsionfree $\Lambda$-module is $R$-torsionfree. Conversely, let $M$ be a finitely generated $R$-torsionfree $\Lambda$-module. Embed $M$ in the $\Gamma$-lattice $\Gamma M$. Then $\Gamma M$ is projective, hence $S_{\Gamma}$-torsionfree, hence $S$-torsionfree, implying that $M$ is $S$-torsionfree. Q.E.D.

REMARK. In geometric language, $\Gamma$ is the normalization of $\Lambda$ (i.e. the integral closure of $\Lambda$ in its total quotient ring $A$ ).

LEMMA 2.3. The R-order $\Lambda$ is a Gorenstein-order.

Proof. The maximal ideal of $\Lambda$ is generated by two elements and it contains the regular element $\pi^{l}$ (by assertion (4) of the previous proof). Therefore the lemma follows from a theorem of Bass [Ba63, Proposition 6.4].

Alternatively, it is easy to give a straightforward and elementary proof of the lemma which is adapted to our specific situation. We present it for convenience of the reader: For each Dynkin diagram $\Delta$ denote the second generating element of $\Lambda$ (in the sense of Lemma 2.1) by $\zeta=\zeta_{\Delta}: \zeta_{\mathbf{A}_{2 m}}=T^{2 m+1}, \zeta_{\mathbf{A}_{2 m-1}}=\left(T^{m},-T^{m}\right), \zeta_{\mathbf{D}_{2 m}}=$ $(0, T, T), \zeta_{\mathbf{D}_{2 m+1}}=\left(0, T^{2}\right), \zeta_{\mathbf{E}_{6}}=T^{4}, \zeta_{\mathbf{E}_{7}}=\left(0, T^{3}\right), \zeta_{\mathbf{E}_{8}}=T^{5}$. Then $\Lambda=k[[\pi, \zeta]] \cong$ $R[Z] /(\varphi(Z))$, the isomorphism being induced by $Z \mapsto \zeta$. Let us specify the polynomials $\varphi(Z)=\varphi_{\Delta}(Z)$ arising in this way: $\varphi_{\mathbf{A}_{n}}(Z)=Z^{2}-\pi^{n+1}, \varphi_{\mathbf{D}_{n}}(Z)=Z^{n-1}-$ $\pi^{2} Z, \quad \varphi_{\mathbf{E}_{6}}(Z)=Z^{3}-\pi^{4}, \quad \varphi_{\mathbf{E}_{7}}(Z)=Z^{3^{n}}-\pi^{3} Z, \quad \varphi_{\mathbf{E}_{8}}(Z)=Z^{3}-\pi^{5}$. Put $d:=\operatorname{deg} \varphi(Z)$ and $\zeta_{i}:=\zeta^{i}, i=0, \ldots, d-1$. Choose the (ordered) set $B=$ $\left\{\zeta_{0}, \ldots, \zeta_{d-1}\right\}$ as canonical $R$-basis of $\Lambda$ and its (ordered) dual basis $B^{*}=$ $\left\{\zeta_{0}^{*}, \ldots, \zeta_{d-1}^{*}\right\}$ as canonical $R$-basis for the dual lattice $\Lambda^{*}=\operatorname{Hom}_{R}(\Lambda, R)$. Then $\zeta$ operates on $\Lambda$ with respect to $B$ by way of the Frobenius-matrix $\phi$ of $\varphi(Z)$, and $\zeta$ operates on $\Lambda^{*}$ with respect to $B^{*}$ by way of ${ }^{t} \phi$, the transpose of $\phi$. Hence $\Lambda$ is a Gorenstein-order if and only if there exists an $R$-basis $B^{\prime}=\left\{\zeta_{0}^{\prime}, \ldots, \zeta_{d-1}^{\prime}\right\}$ of $\Lambda$ such that $\zeta$ operates on $\Lambda$ with respect to $B^{\prime}$ by way of ${ }^{t} \phi$. Indeed, there exists an $R$-basis $B^{\prime}$ of $\Lambda$ with this property: If $\varphi(Z)=Z^{d}-a$ (the cases $\mathbf{A}_{n}, \mathbf{E}_{6}, \mathbf{E}_{8}$ ), then put $\zeta_{i}^{\prime}:=\zeta_{d-1-i}$ for all $i=0, \ldots, d-1$. If $\varphi(Z)=Z^{d}-a Z$ (the cases $\mathbf{D}_{n}, \mathbf{E}_{7}$ ), then put $\zeta_{0}^{\prime}:=\zeta_{d-1}-a \zeta_{0}$ and $\zeta_{i}^{\prime}:=\zeta_{d-1-i}$ for all $i=1, \ldots, d-1$. Q.E.D.

In view of Lemmata 2.2 and 2.3 the whole theory of lattices over (Gorenstein)orders is at our disposal. In the following corollaries we collect as much information from this background as will be needed in $\S 3$ for the construction of the AuslanderReiten quiver of $\Lambda$.

Denote by ind $\Lambda$ the full subcategory of $\mathscr{S}_{\Lambda}$ which consists of all indecomposable $\Lambda$-lattices. For any $M \in \mathscr{S}_{\Lambda}$, denote by $[M]$ the isomorphism class of $M$. For definitions of Auslander-Reiten sequence and Auslander-Reiten translation $\tau$ see [Ro81].

COROllary 2.4. (i) For each $M \in$ ind $\Lambda \backslash[\Lambda]$ there exists an Auslander-Reiten sequence starting in $M$ and an Auslander-Reiten sequence ending in $M$.

(ii) The Auslander-Reiten translation $\tau$ coincides with Heller's loop space operator $\Omega$. 
(iii) Let $M \in$ ind $\Lambda \backslash[\Lambda]$ and $\mathscr{E}: 0 \rightarrow \tau M \rightarrow E \rightarrow M \rightarrow 0$ be a short exact sequence. Then $\mathscr{E}$ is an Auslander-Reiten sequence if and only if $\mathscr{E}$ represents a nonzero element in the simple socle of $\operatorname{Ext}_{\Lambda}^{1}(M, \tau M)$, viewed as right End $M$-module or as left End $\tau M$-module.

(iv) Let $0 \rightarrow M \stackrel{\psi}{\rightarrow} E \stackrel{\psi^{\prime}}{\rightarrow} M^{\prime} \rightarrow 0$ be an Auslander-Reiten sequence in $\mathscr{S}_{\Lambda}, E=$ $\oplus_{i=1}^{m} E_{i}$ be the decomposition of $E$ into indecomposable direct summands, $\psi=$ $\left(\psi_{i}\right)_{i=1, \ldots, m}$ and $\psi^{\prime}=\left(\psi_{i}^{\prime}\right)_{i=1, \ldots, m}$ be the corresponding decompositions of $\psi$ and $\psi^{\prime}$. Then $\psi_{i}: M \rightarrow E_{i}$ and $\psi_{i}^{\prime}: E_{i} \rightarrow M^{\prime}$ are irreducible maps for all $i=1, \ldots, m$. Conversely, let $X, X^{\prime} \in$ ind $\Lambda$ and $\xi: M \rightarrow X, \xi^{\prime}: X^{\prime} \rightarrow M^{\prime}$ be irreducible maps. Then there exist isomorphisms $\varphi_{i}: E_{i} \rightarrow X, \varphi_{j}^{\prime}: X^{\prime} \rightarrow E_{j}, i, j \in\{1, \ldots, m\}$, such that $\xi=\varphi_{i} \psi_{i}$ and $\xi^{\prime}=\psi_{j}^{\prime} \varphi_{j}^{\prime}$.

(v) Let $J$ be the Jacobson radical of $\Lambda$ and $J^{*}$ the unique minimal over-order of $\Lambda$. Assume that $\Delta \neq \mathbf{A}_{1}$. Then $J, J^{*} \in$ ind $\Lambda$ and the inclusions $\iota: J \hookrightarrow \Lambda, \iota^{*}: \Lambda \hookrightarrow J^{*}$ are irreducible maps. Conversely, let $X, X^{\prime} \in$ ind $\Lambda$ and $\xi: X \rightarrow \Lambda, \xi^{\prime}: \Lambda \rightarrow X^{\prime}$ be irreducible maps. Then there exist isomorphisms $\varphi: X \rightarrow J, \varphi^{\prime}: J^{*} \rightarrow X^{\prime}$ such that $\xi=\iota \varphi$ and $\xi^{\prime}=\varphi^{\prime} \iota^{*}$.

(vi) If $\mathscr{B}$ is a finite connected component of $\mathscr{A}(\Lambda)$, then $\mathscr{B}=\mathscr{A}(\Lambda)$.

Proof. From the embedding $\Lambda \subset \Gamma$ it is easy to see that $J^{*}$ is local if $\Delta \neq \mathbf{A}_{1}$. Hence $J^{*}$ is an indecomposable $\Lambda$-lattice and, by duality, $J$ is an indecomposable $\Lambda$-lattice. Now all statements emerge from standard Auslander-Reiten theory when applied to a local Gorenstein-order with indecomposable radical (see [Au75, Ro77, Ro81]). Q.E.D.

Besides Auslander-Reiten theory we shall need the reduction functor which was introduced in [Gr/Re78 and Rin/Ro79]: For each Dynkin diagram $\Delta$ let $I$ be the maximal $\Gamma$-ideal contained in $\Lambda$. Put $\bar{\Lambda}:=\Lambda / I, \bar{\Gamma}:=\Gamma / I=\oplus_{i=1}^{r} \bar{\Gamma} e_{i}$, and denote by $\bmod \bar{\Lambda}$ and mod $\bar{\Gamma}$ the categories of finitely generated $\bar{\Lambda}$-modules and finitely generated $\bar{\Gamma}$-modules. Consider the category $\mathscr{C}=\mathscr{C}_{\Delta}$ which is defined as follows: Objects in $\mathscr{C}$ are triples $(U, V, \iota)$, where $U \in \bmod \bar{\Lambda}, V \in \bmod \bar{\Gamma}, V$ is $\bar{\Gamma}$-projective, $\iota: U \rightarrow V$ is a $\bar{\Lambda}$-monomorphism and $\bar{\Gamma}(\operatorname{im} \iota)=V$. Morphisms in $\mathscr{C}$ from $(U, V, \iota)$ to $\left(U^{\prime}, V^{\prime}, \iota^{\prime}\right)$ are pairs $(\alpha, \beta)$, where $\alpha \in \operatorname{Hom}_{\bar{\Lambda}}\left(U, U^{\prime}\right), \beta \in \operatorname{Hom}_{\bar{\Gamma}}\left(V, V^{\prime}\right)$ and $\beta \iota=$ $\iota^{\prime} \alpha$. The categories $\mathscr{S}_{\Lambda}$ and $\mathscr{C}$ are related by the functor $\mathscr{F}: \mathscr{S}_{\Lambda} \rightarrow \mathscr{C}$, given by $\mathscr{F}(M)=\left(M / M I, M \Gamma / M I, \iota /\left.\iota\right|_{M I}\right), \iota: M \hookrightarrow M \Gamma$ being the inclusion map.

COROllaRY 2.5. The functor $\mathscr{F}: \mathscr{S}_{\Lambda} \rightarrow \mathscr{C}$ is a representation equivalence.

Proof. Because $\mathscr{S}_{\Lambda}$ is the category of $\Lambda$-lattices and $\Gamma$ is the maximal order in $A$, the statement follows from [Gr/Re78 and $\mathbf{R i} / \mathbf{R o} 79]$. Q.E.D.

Our next step is to combine the two concepts described in Corollaries 2.4 and 2.5. Define mappings $\operatorname{dim}^{\prime}: \mathscr{S}_{\Lambda} \rightarrow \mathbf{Z}^{(r)}$ and $\operatorname{dim}: \mathscr{S}_{\Lambda} \rightarrow \mathbf{Z} \oplus \mathbf{Z}^{(r)}$ by $\operatorname{dim}^{\prime}(M)=$ $\left(\operatorname{dim}_{k}\left(M \Gamma e_{i} / M I e_{i}\right) \overline{i=1, \ldots, r}_{i}\right.$ and $\left.\operatorname{dim}(M)=\overline{\left(\operatorname{dim}_{k}\right.}(M / M I), \operatorname{dim}^{\prime}(M)\right)$. We call $\operatorname{dim}$ the dimension-mapping on $\mathscr{S}_{\Lambda}$, an $\overline{\operatorname{dim}}(M)$ the dimension-type of $M$.

Lemma 2.6. Let $0 \rightarrow M \rightarrow E \rightarrow M^{\prime} \rightarrow 0$ be an Auslander-Reiten sequence in $\mathscr{S}_{\Lambda}$. Then:

(i) $\operatorname{dim}^{\prime}(E)=\operatorname{dim}^{\prime}(M)+\operatorname{dim}^{\prime}\left(M^{\prime}\right)$.

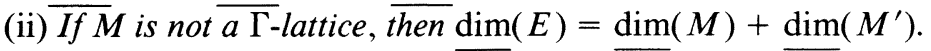


Proof. Since $\Gamma$ is the maximal order in $A, \operatorname{dim}^{\prime}(M)$ depends only on the isomorphism class of $K M$ as $A$-module. Therefore statement (i) is true for any short exact sequence in $\mathscr{S}_{\Lambda}$.

For proving (ii), consider the following commutative diagram:

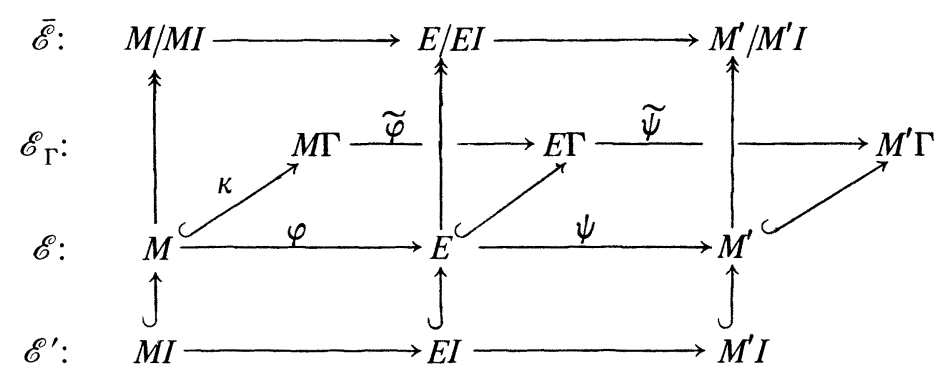

Let us show that $\mathscr{E}_{\Gamma}$ is a split short exact sequence: Clearly $\tilde{\varphi}$ is a monomorphism, $\tilde{\psi}$ is an epimorphism and $\operatorname{im} \tilde{\varphi} \subset \operatorname{ker} \tilde{\psi}$. Because $\kappa$ is not a splittable monomorphism (by assumption) there exists a $\Lambda$-homomorphism $\xi: E \rightarrow M \Gamma$ which extends to a retraction $\tilde{\xi}: E \Gamma \rightarrow M \Gamma$ (due to the factorization property of the Auslander-Reiten sequence $\mathscr{E}$ ). Hence $E \Gamma=\operatorname{im} \tilde{\varphi} \oplus \operatorname{ker} \tilde{\xi} \subset \operatorname{ker} \tilde{\psi}+\operatorname{ker} \tilde{\xi}$. However, ker $\tilde{\psi} \cap \operatorname{ker} \tilde{\xi}=$ 0 , implying that $\operatorname{im} \tilde{\varphi}=\operatorname{ker} \tilde{\psi}$. Therefore $\mathscr{E}_{\Gamma}$ is split short exact.

As a consequence, $\mathscr{E}^{\prime}$ is a split short exact sequence of $\Gamma$-lattices and $\overline{\mathscr{E}}$ is a short exact sequence of $\bar{\Lambda}$-modules. Q.E.D.

3. Proof of Theorem A (second part): Construction of $\mathscr{A}(\Lambda)$. We keep the notation of the previous section and fix some additional conventions: Denote by $J$ the Jacobson radical of $\Lambda$ and by $J^{*}$ the unique minimal over-order of $\Lambda$. Denote by ${ }_{M} \mathscr{E}$ the Auslander-Reiten sequence with starting term $M$ and by $\mathscr{E}_{M}$ the Auslander-Reiten sequence with end term $M$. The full subquiver of $\mathscr{A}(\Lambda)$ which corresponds to a single Auslander-Reiten sequence is called a "mesh". In particular ${ }_{J} \mathscr{E}$ gives rise to the "projective mesh".

If there is need to describe a $\Lambda$-lattice $M$ explicitly, then we shall do this in terms of the embedding $M \subset \Gamma M \cong \oplus_{i=1}^{r} \Gamma_{i}^{\left(n_{i}\right)}$ (where $\Gamma_{i}:=\Gamma e_{i}$, for the sake of brevity). We label the indecomposable $\Lambda$-lattices according to their dimension-types (i.e. if we write $M=M(\underline{d}), \underline{d} \in \mathbf{Z} \oplus \mathbf{Z}^{(r)}$, then $\left.\underline{d}=\operatorname{dim} M\right)$. Nonisomorphic indecomposable $\Lambda$-lattices which have the same dimension-type will be distinguished by dashes.

In this section we construct explicitly the Auslander-Reiten quiver $\mathscr{A}(\Lambda)$ for each Dynkin diagram $\Delta$. In each case the strategy will be the following: Start with the meshes $\mathscr{E}_{J^{*}}$ and $\mathscr{E}_{J}$ (usually these are easily derived from the given embedding $\Lambda \subset \Gamma$ ). Continue by "completing meshes", using the additivity of the dimensionmapping (Lemma 2.6). This procedure works algorithmically as long as we can be sure that the dimension-type which completes a mesh belongs to an indecomposable $\Lambda$-lattice. This is in some cases immediately clear from the embedding $\bar{\Lambda} \subset \bar{\Gamma}$, in 
other cases it can be derived from the knowledge of a few distinguished AuslanderReiten sequences which have to be calculated directly. On the other hand, in those places where an Auslander-Reiten sequence has three middle terms, the above algorithm fails. More direct calculation is necessary in order to deal with such situations.

In the following propositions we describe the Auslander-Reiten quiver $\mathscr{A}(\Lambda)$ for each Dynkin diagram $\Delta$ in terms of its universal covering $\tilde{\mathscr{A}}(\Lambda)$ together with an automorphism $\tilde{\alpha}$ of $\tilde{\mathscr{A}}(\Lambda)$, such that $\mathscr{A}(\Lambda) \cong \tilde{\mathscr{A}}(\Lambda) / \tilde{\alpha} \mathbf{Z}$. In each case the description of $\tilde{\alpha}$ involves the automorphism $\tilde{\tau}$ which is understood to be the unique shift to the left such that $(\tilde{\mathscr{A}}(\Lambda), \tilde{\tau})$ is a translation quiver. In place of the points of $\tilde{\mathscr{A}}(\Lambda)$ we note the dimension-type of the corresponding points of $\mathscr{A}(\Lambda)$ (using dashes if necessary in order to distinguish different points which have the same dimensiontype).

The presentation of the proofs will be such that first we collect all material which has to be worked out directly, and then we give a brief sketch of how to construct $\mathscr{A}(\Lambda)$ on the basis of this information.

Note that, once Propositions 3.1-3.7 are proved, a complete list of all indecomposable $\Lambda$-lattices is easily obtained. Namely, let $n(\underline{d})$ be the number of points in $\mathscr{A}(\Lambda)$ which have dimension-type $\underline{d}$ and let $D=\left\{\underline{d} \in \mathbf{Z} \oplus \mathbf{Z}^{(r)} \mid n(\underline{d})>0\right\}$. For each $\underline{d} \in D$ specify $\Lambda$-lattices $M(\underline{d})_{1}, \ldots, M(\underline{d})_{n(\underline{d})}$ which are indecomposable, pairwise nonisomorphic, and all of which have dimension-type $\underline{d}$. (This is an easy exercise.) Then, in view of Propositions 3.1-3.7, it is clear that

$$
\bigcup_{\underline{d} \in D}\left\{M(\underline{d})_{1}, \ldots, M(\underline{d})_{n(\underline{d})}\right\}
$$

is a complete set of representatives for the isomorphism classes of indecomposable $\Lambda$-lattices. The reader who is curious to see this list explicitly is referred to [Gr/Kn85].

Finally, let us mention that there is a close connection between some of the complete local rings $\Lambda_{\Delta}$ and certain group rings which arise in integral representation theory of finite groups, in the sense that their Auslander-Reiten quivers are isomorphic. To be precise, let $L$ be a complete discrete valuation ring with exponential valuation $v$, and let $C_{n}$ be the cyclic group of order $n$. Then the following are true:

(i) If $\Delta=\mathbf{A}_{1}$ and $v(p)=1$, then $\mathscr{A}\left(\Lambda_{\Delta}\right) \cong \mathscr{A}\left(L C_{p}\right)$ for all prime numbers $p$.

(ii) If $\Delta=\mathbf{A}_{2 m-1}$ and $v(2)=m$, then $\mathscr{A}\left(\Lambda_{\Delta}\right) \cong \mathscr{A}\left(L C_{2}\right)$ for all $m \in \mathbf{N}$.

(iii) If $\Delta=\mathbf{D}_{2 p}$ and $v(p)=1$, then $\mathscr{A}\left(\Lambda_{\Delta}\right) \cong \mathscr{A}\left(L C_{p^{2}}\right)$ for all prime numbers $p$.

(iv) If $\Delta=\mathbf{D}_{p+1}$ and $v(p)=2$ and the $p$ th cyclotomic polynomial is reducible over $L$, then $\mathscr{A}\left(\Lambda_{\Delta}\right) \cong \mathscr{A}\left(L C_{p}\right)$ for all prime numbers $p>2$.

(v) If $\Delta=\mathbf{E}_{7}$ and $v(3)=3$, then $\mathscr{A}\left(\Lambda_{\Delta}\right) \cong \mathscr{A}\left(L C_{3}\right)$.

The Auslander-Reiten quivers of the group rings mentioned above are well known (see [Bu/79] for case (ii), [Wi82] for case (iii) and [Di83] for a unified treatment of cases (i)-(v)). Hence the asserted isomorphisms of Auslander-Reiten quivers will become evident from Propositions 3.2, 3.3 and 3.6. 


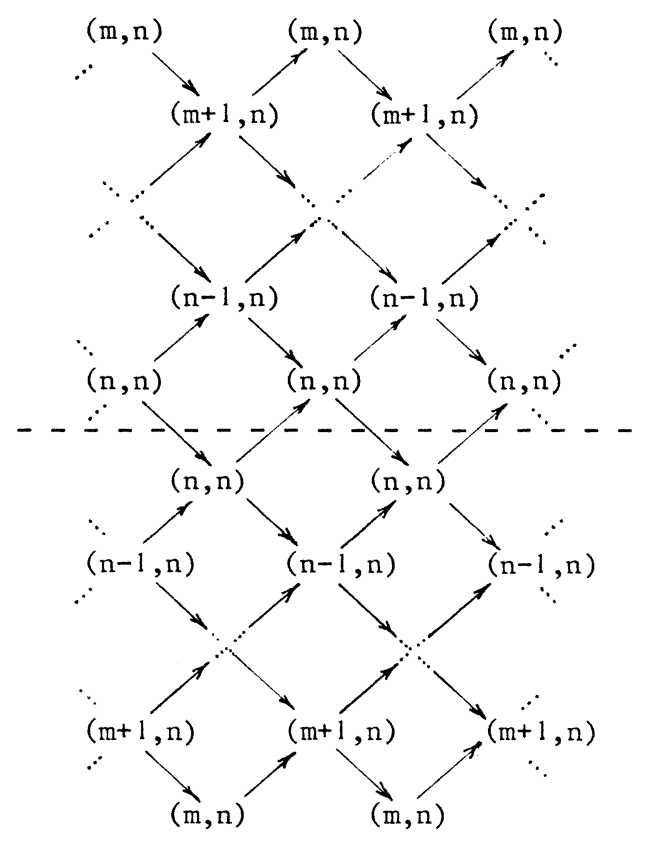

FIGURE 2

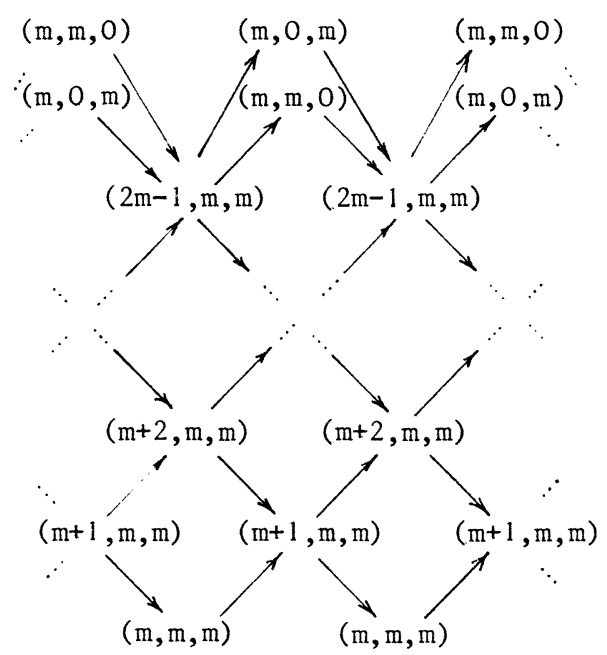

Figure 3

Proposition 3.1. Let $\Delta=\mathbf{A}_{n}, n=2 m, m \in \mathbf{N}$. Then $\mathscr{A}\left(\Lambda_{\Delta}\right)$ is given by Figure 2 where one has to identify modulo $\tilde{\tau} / 2+\tilde{\sigma}$, $\tilde{\sigma}$ being the reflection with respect to the dotted horizontal axis.

Proof. Verify directly:

(1) $\underline{\operatorname{dim}} \Lambda=(m, n), \underline{\operatorname{dim}} J=(m+1, n), J \cong J^{*}$. 
(2) The Auslander-Reiten sequence $\mathscr{E}_{\Gamma}$ is given by the commutative diagram

$$
\begin{aligned}
& \begin{array}{llcccccc} 
& \rightarrow & \stackrel{\tilde{\varphi}}{\rightarrow} & \Gamma \oplus \Gamma & \stackrel{\tilde{\psi}}{\rightarrow} & \Gamma & \rightarrow & 0 \\
& & \uparrow & & \|
\end{array} \\
& \mathscr{E}_{\Gamma}: \quad 0 \quad \rightarrow \quad \Gamma \stackrel{\varphi}{\rightarrow} \Gamma \oplus\left(\Lambda+T^{3} \Lambda\right) \stackrel{\psi}{\rightarrow} \Gamma \quad \Gamma \quad \rightarrow \quad 0
\end{aligned}
$$

where $\tilde{\varphi}=\left(\begin{array}{c}T \\ T^{2}\end{array}\right), \tilde{\psi}=(-T 1)$.

Begin the construction of $\mathscr{A}(\Lambda)$ with the projective mesh (using (1)). Continue inductively, using the additivity of the dimension-mapping, up to $\mathscr{E}_{M(n-1, n)}$. Complete the construction with $\mathscr{E}_{\Gamma}$ (using (2)). Q.E.D.

Proposition 3.2. Let $\Delta=\mathbf{A}_{n}, n=2 m-1, m \in \mathbf{N}$. Then $\mathscr{A}\left(\Lambda_{\Delta}\right)$ is given by Figure 3 where one has to identify modulo $\tilde{\tau}+\tilde{\rho}, \tilde{\rho}$ being the automorphism which exchanges $(m, m, 0)$ and $(m, 0, m)$.

Proof. Verify directly:

(1) $\operatorname{dim} \Lambda=(m, m, m), \operatorname{dim} J=(m+1, m, m), J \cong J^{*}$.

(2) If $\operatorname{dim} M=(l, m, m)$ and $l<2 m$, then $\mathscr{F} M$ is indecomposable, hence $M$ is indecomposable. (This follows easily from the structure of $\bar{\Lambda} \subset \bar{\Gamma}$.)

If $n=1$, then $J=\Gamma_{1} \oplus \Gamma_{2}$ and there are exactly three isomorphism classes of indecomposable $\Lambda$-lattices, represented by $\left\{\Lambda, \Gamma_{1}, \Gamma_{2}\right\}$.

If $n>1$, then begin the construction of $\mathscr{A}(\Lambda)$ with the projective mesh (using (1)). Continue inductively, using the additivity of the dimension-mapping and (2), until the construction terminates with $\mathscr{E}_{\Gamma_{1}}$ and $\mathscr{E}_{\Gamma_{2}}$. Q.E.D.

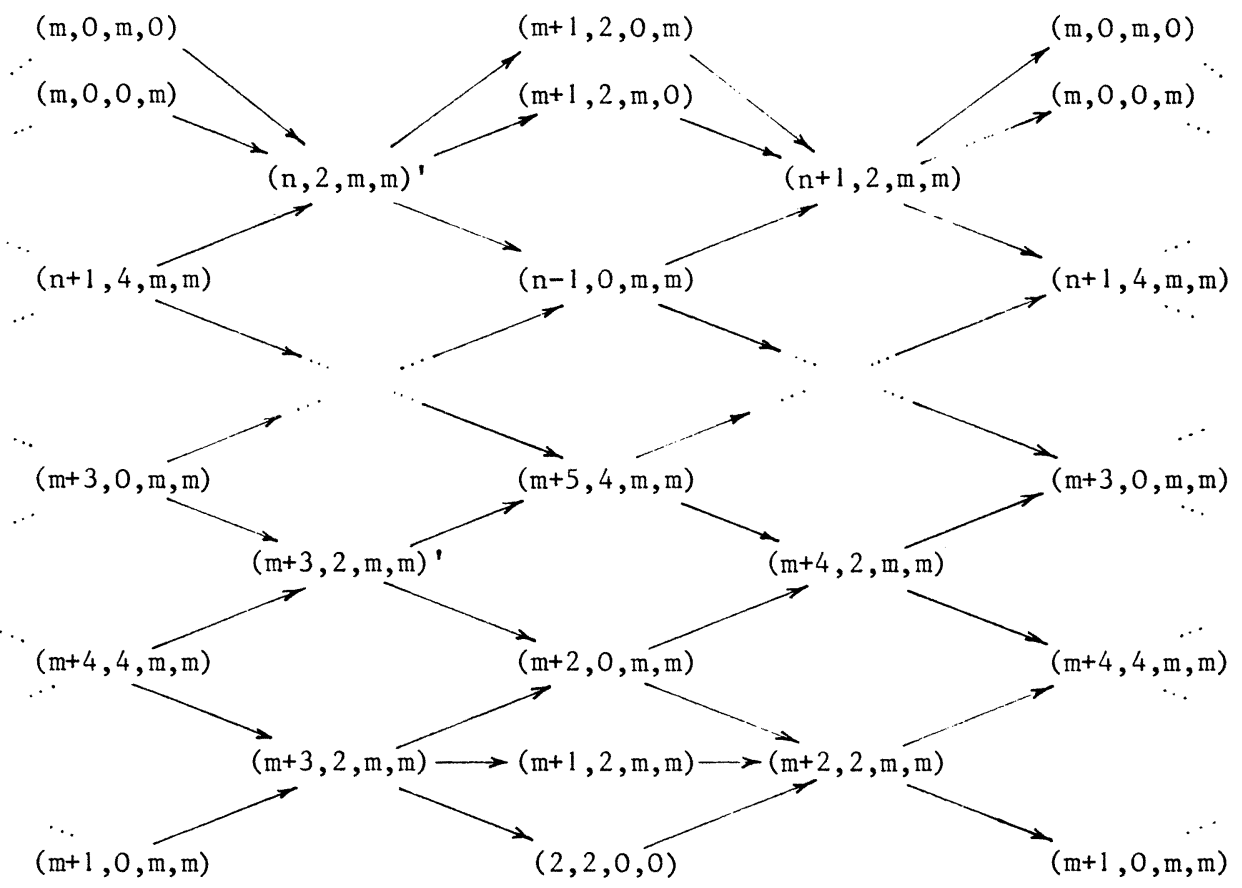

FigURE 4 
Proposition 3.3. Let $\Delta=\mathbf{D}_{n}, n=2 m, m \in \mathbf{N} \backslash\{1\}$. Then $\mathscr{A}\left(\Lambda_{\Delta}\right)$ is given by Figure 4 where one has to identify modulo $2 \tilde{\tau}$.

Proof. Verify directly:

(1) $\tau\left(\Gamma_{1}\right) \cong(1,1) \Lambda \subset \Gamma_{2} \oplus \Gamma_{3} ; \quad \tau\left(\left(e_{2}+e_{3}\right) \Lambda\right) \cong \Gamma_{1} ; \operatorname{dim}\left(\left(e_{2}+e_{3}\right) \Lambda\right)=$ $(m+1,0, m, m) ; \operatorname{dim} \Gamma_{1}=(2,2,0,0)$. The Auslander-Reiten sequence $\mathscr{E}_{\Gamma_{1}}$ is given by the commutative diagram

$$
\begin{aligned}
& 0 \quad \rightarrow \quad \Gamma_{2} \oplus \Gamma_{3} \quad \stackrel{\tilde{\varphi}}{\rightarrow} \quad \Gamma_{1} \oplus \Gamma_{2} \oplus \Gamma_{3} \quad \stackrel{\tilde{\psi}}{\rightarrow} \Gamma_{1} \quad \rightarrow \quad 0 \\
& \uparrow \quad \uparrow \quad \uparrow \\
& \mathscr{E}_{\Gamma_{1}}: 0 \quad 0 \quad \rightarrow \quad(T, T) \Lambda \stackrel{\varphi}{\rightarrow} \quad J \quad \stackrel{\psi}{\rightarrow} T \Gamma_{1} \quad \rightarrow \quad 0
\end{aligned}
$$

where $\tilde{\varphi}$ is the canonical inclusion and $\tilde{\psi}$ is the canonical projection.

(2) $\operatorname{dim} \Lambda=(m+1,2, m, m), \underline{\operatorname{dim}} J=(m+3,2, m, m), \underline{\operatorname{dim}} J^{*}=$ $(m+2, \overline{2,} m, m), \tau(J)=J^{*}$.

(3) If $\operatorname{dim} M=(l, 0, m, m)$ and $l<2 m$, then $M$ is indecomposable.

(4) Let $M$ be a direct summand of the middle term of $\mathscr{E}_{X}, X \not J$. If $\operatorname{dim} M=(l, 2, m, m)$ and $l \leqslant 2 m$, then $M$ is indecomposable.

(5)

$$
\begin{aligned}
& \tau\left(\Gamma_{2}\right) \cong M(m+1,2,0, m):=\left(T, T^{m-1}\right) \Lambda \subset \Gamma_{1} \oplus \Gamma_{3}, \\
& \tau\left(\Gamma_{3}\right) \cong M(m+1,2, m, 0):=\left(T, T^{m-1}\right) \Lambda \subset \Gamma_{1} \oplus \Gamma_{2} .
\end{aligned}
$$

Begin the construction of $\mathscr{A}(\Lambda)$ with $\mathscr{E}_{\Gamma_{1}},{ }_{J}^{\mathscr{E}}, \Gamma_{1} \mathscr{E}$ and $\mathscr{E}_{J}$ (using (1), (2) and (3)). Continue inductively, stepping from $\tau$-orbit to $\tau$-orbit by way of completing the meshes of $\mathscr{A}(\Lambda)$, using the additivity of the dimension-mapping. Observe that in this procedure the dimension-type which completes a mesh always belongs to an indecomposable lattice (in view of (3) and (4)), until one reaches the $\tau$-orbit of $M(n+1,2, m, m)$. Now the construction terminates (using (5)). Q.E.D.

Proposition 3.4. Let $\Delta=\mathbf{D}_{n+1}, n=2 m, m \in \mathbf{N} \backslash\{1\}$. Then $\mathscr{A}\left(\Lambda_{\Delta}\right)$ is given by Figure 5 where one has to identify modulo $\tilde{\tau}+\tilde{\sigma}$, $\tilde{\sigma}$ being the reflection with respect to the dotted horizontal axis.

Proof. Verify directly:

(1) $\tau\left(\Gamma_{1}\right) \cong e_{2} \Lambda \subset \Gamma_{2} ; \tau\left(e_{2} \Lambda\right) \cong \Gamma_{1}, \operatorname{dim}\left(e_{2} \Lambda\right)=(m+1,0, n) ; \operatorname{dim} \Gamma_{1}=(2,2,0)$. The Auslander-Reiten sequence $\mathscr{E}_{\Gamma_{1}}$ is given by the commutative diagram

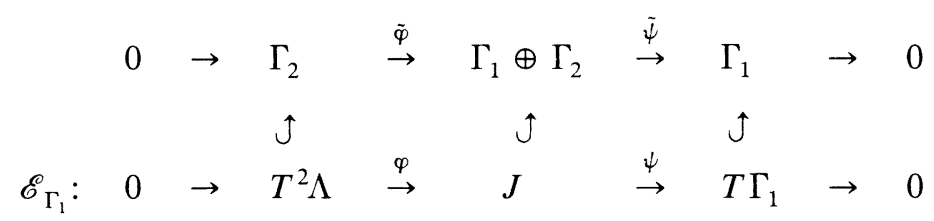

where $\tilde{\varphi}$ is the canonical inclusion and $\tilde{\psi}$ is the canonical projection.

(2) $\operatorname{dim} \Lambda=(m+1,2, n), \underline{\operatorname{dim}} J=(m+3,2, n), \underline{\operatorname{dim}} J^{*}=(m+2,2, n)$, $\tau(J) \cong \overline{J^{*}}$. 
(3) Let $M$ be a direct summand of the middle term of ${ }_{x} \mathscr{E}, X \not J$. If $\operatorname{dim} M=(l, 2, n)$, then $M$ is indecomposable.

(4) The following $\Lambda$-lattices are indecomposable:

$$
\begin{aligned}
& M(n+1,2, n)=\Gamma_{2}(0, T)+(1,1) \Lambda \subset \Gamma_{1} \oplus \Gamma_{2}, \\
& M(n+2,4, n)=\left(\Gamma_{1}^{(2)} \oplus \Gamma_{2}\right)\left(T, T^{2}\right)+(0,1,1) \Lambda+(1,0, T) \Lambda \subset \Gamma_{1}^{(2)} \oplus \Gamma_{2} .
\end{aligned}
$$

(5) The Auslander-Reiten sequence $\mathscr{E}_{M(n+1,2, n)}$ is given by the commutative diagram

$$
\begin{array}{cccccc}
0 & \rightarrow \quad \Gamma_{1} \oplus \Gamma_{2} & \stackrel{\tilde{\varphi}}{\rightarrow}\left(\Gamma_{1}^{(2)} \oplus \Gamma_{2}\right) \oplus \Gamma_{2} & \stackrel{\dot{\psi}}{\rightarrow} \Gamma_{1} \oplus \Gamma_{2} & \rightarrow 0 \\
& \uparrow & & \mathcal{J} & & \mathcal{\jmath} \\
\mathscr{E}_{M(n+1,2, n)}: & 0 & \rightarrow M(n+1,2, n) & \stackrel{\varphi}{\rightarrow} M(n+2,4, n) \oplus \Gamma_{2} & \stackrel{\psi}{\rightarrow} M(n+1,2, n) & \rightarrow 0
\end{array}
$$

where

$$
\tilde{\varphi}=\left(\begin{array}{cc}
0 & 0 \\
1 & 0 \\
0 & T \\
\hline 0 & 1
\end{array}\right), \quad \tilde{\psi}=\left(\begin{array}{ccc|c}
1 & 0 & 0 & 0 \\
0 & 0 & 1 & -T
\end{array}\right)
$$

Now the construction of $\mathscr{A}(\Lambda)$ works as in the previous case, up to the $\tau$-orbit of $\Gamma_{2}$. Use (5) in order to complete the construction. Q.E.D.

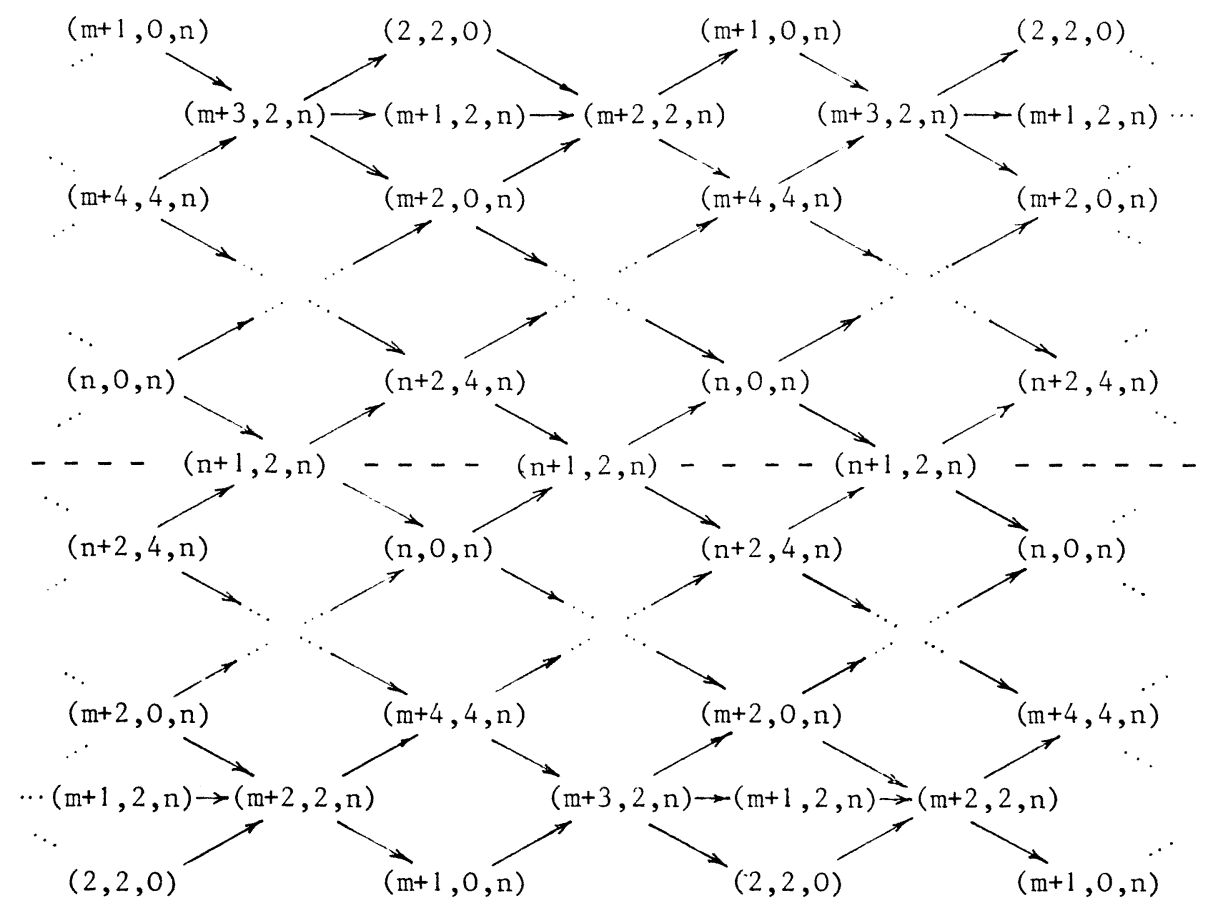

FigURE 5 


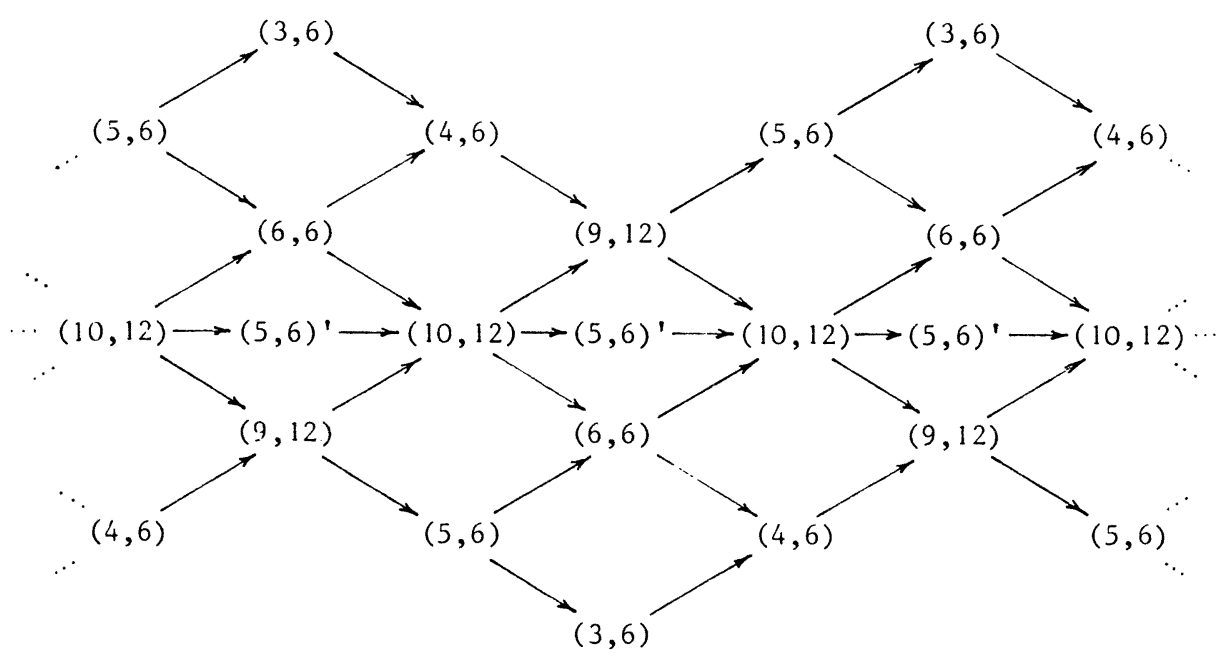

Figure 6

Proposition 3.5. Let $\Delta=\mathbf{E}_{6}$. Then $\mathscr{A}\left(\Lambda_{\Delta}\right)$ is given by Figure 6 where one has to identify modulo $\tilde{\tau}+\tilde{\sigma}, \tilde{\sigma}$ being the reflection with respect to the horizontal axis which connects $(5,6)^{\prime}$ and $(10,12)$.

Proof. Verify directly:

(1) $\operatorname{dim} \Lambda=(3,6), \underline{\operatorname{dim}} J=(5,6), \underline{\operatorname{dim}} J^{*}=(4,6)$.

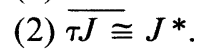

(3) The following $\Lambda$-lattices are indecomposable:

$$
\begin{aligned}
& M(10,12)=\left[\left(\Lambda+T^{2} \Lambda\right) \oplus\left(\Lambda+T^{5} \Lambda\right)\right]+\left(T, T^{2}\right) \Lambda \subset \Gamma^{(2)}, \\
& M(9,12)=\left[\left(\Lambda+T^{5} \Lambda\right) \oplus\left(\Lambda+T^{5} \Lambda\right)\right]+\left(T, T^{2}\right) \Lambda \subset \Gamma^{(2)} \\
& M(5,6)^{\prime}=\Lambda+T^{2} \Lambda \subset \Gamma
\end{aligned}
$$

(4) The Auslander-Reiten sequence $\mathscr{E}_{M(10,12)}$ is given by the commutative diagram

$$
\begin{aligned}
& \begin{array}{ccccccc}
0 \rightarrow & \Gamma^{(2)} & \stackrel{\tilde{\varphi}}{\rightarrow} & \Gamma^{(2)} \oplus \Gamma \oplus \Gamma & \stackrel{\tilde{\psi}}{\rightarrow} & \Gamma^{(2)} & \rightarrow 0 \\
\uparrow & & \uparrow & & \uparrow &
\end{array} \\
& \mathscr{E}_{M(10,12)}: \quad 0 \quad \rightarrow M(10,12) \quad \stackrel{\varphi}{\rightarrow} M(9,12) \oplus M(5,6)^{\prime} \oplus \Gamma \quad \stackrel{\psi}{\rightarrow} M(10,12) \quad \rightarrow 0
\end{aligned}
$$

where

$$
\tilde{\varphi}=\left(\begin{array}{ll}
T & -1 \\
T^{2} & 0 \\
\hline 0 & 1 \\
\hline-1 & 0
\end{array}\right), \quad \tilde{\psi}=\left(\begin{array}{ll|l|l}
1 & 0 & 1 & T \\
0 & 1 & 0 & T^{2}
\end{array}\right)
$$

Begin the construction of $\mathscr{A}(\Lambda)$ with the projective mesh (using (1)). Continue with $\mathscr{E}_{J}$ (using (2)). Complete the construction in view of (3) and (4). Q.E.D. 


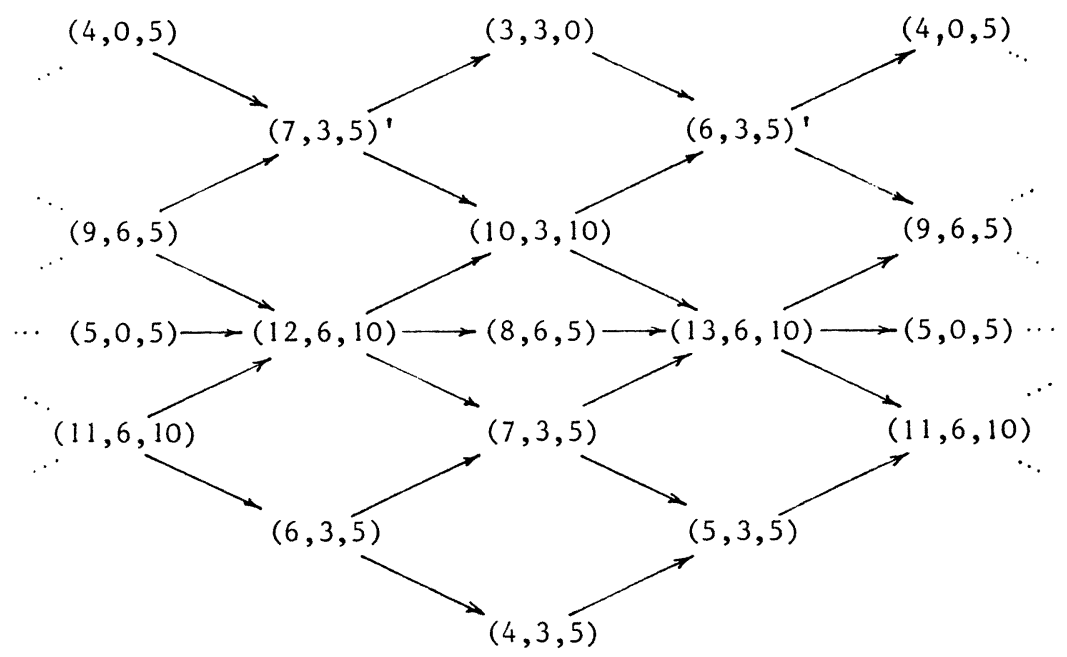

FIGURE 7

Proposition 3.6. Let $\Delta=\mathbf{E}_{7}$. Then $\mathscr{A}\left(\Lambda_{\Delta}\right)$ is given by Figure 7 where one has to identify modulo $2 \tilde{\tau}$.

Proof. Verify directly:

(1) $\operatorname{dim} \Lambda=(4,3,5), \operatorname{dim} J=(6,3,5), \operatorname{dim} J^{*}=(5,3,5), \tau(J) \cong J^{*}$.

(2) $\bar{T}$ he following $\Lambda$-lattices are indecomposable:

$$
\begin{aligned}
M(11,6,10)= & \Gamma_{2}^{(2)}\left(0, T^{3}\right)+(1,0,1,0) \Lambda+(0,1,0,1) \Lambda+(T, 0,0, T) \Lambda \\
\subset & \Gamma_{1}^{(2)} \oplus \Gamma_{2}^{(2)}, \\
M(13,6,10)= & {\left[\Gamma_{2}\left(0, T^{3}\right) \oplus \Gamma_{2}(0, T)\right]+(1,0,0,1) \Lambda } \\
& +(0,1,1,0) \Lambda+(1,0, T, 0) \Lambda \subset \Gamma_{1}^{(2)} \oplus \Gamma_{2}^{(2)}, \\
M(7,3,5)= & \Gamma_{2}(0, T)+(1,1) \Lambda \subset \Gamma_{1} \oplus \Gamma_{2}, \\
M(8,6,5)= & \Gamma_{2}\left(0, T^{3}\right)+(0,1,1) \Lambda+(1,0, T) \Lambda \subset \Gamma_{1}^{(2)} \oplus \Gamma_{2}, \\
M(7,3,5)^{\prime}= & (0,1) \Lambda+(1, T) \Lambda \subset \Gamma_{1} \oplus \Gamma_{2}, \\
M(4,0,5)= & e_{2} \Lambda \subset \Gamma_{2} .
\end{aligned}
$$

(3) The Auslander-Reiten sequences $\mathscr{E}_{M(11,6,10)}, \mathscr{E}_{\Gamma_{2}}, \mathscr{E}_{\Gamma_{1}}$ are given by the following commutative diagrams:

$$
\begin{aligned}
& 0 \quad \rightarrow \Gamma_{1} \oplus \Gamma_{2} \quad \stackrel{\tilde{\varphi}}{\rightarrow}\left(\Gamma_{1}^{(2)} \oplus \Gamma_{2}^{(2)}\right) \oplus\left(\Gamma_{1} \oplus \Gamma_{2}\right) \stackrel{\tilde{\psi}}{\rightarrow} \Gamma_{1}^{(2)} \oplus \Gamma_{2}^{(2)} \quad \rightarrow 0
\end{aligned}
$$

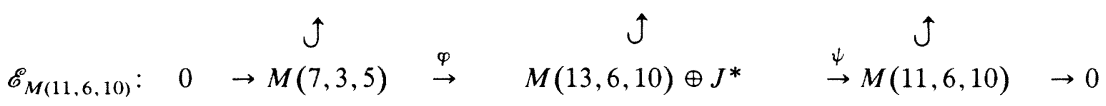


where

$$
\begin{aligned}
& \tilde{\varphi}=\left(\begin{array}{cc}
1 & 0 \\
0 & 0 \\
0 & 0 \\
0 & 1 \\
\hline T & 0 \\
0 & T^{2}
\end{array}\right), \quad \tilde{\psi}=\left(\begin{array}{cccc|cc}
T & 0 & 0 & 0 & -1 & 0 \\
0 & 1 & 0 & 0 & 0 & 0 \\
0 & 0 & 0 & T^{2} & 0 & -1 \\
0 & 0 & 1 & 0 & 0 & 0
\end{array}\right) \\
& 0 \quad \rightarrow \quad \Gamma_{1}^{(2)} \oplus \Gamma_{2} \quad \stackrel{\tilde{\varphi}}{\rightarrow} \Gamma_{1}^{(2)} \oplus \Gamma_{2}^{(2)} \quad \stackrel{\dot{\psi}}{\rightarrow} \quad \Gamma_{2} \quad \rightarrow \quad 0
\end{aligned}
$$

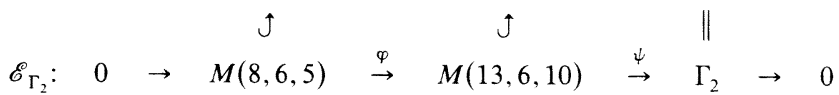

where

$$
\begin{aligned}
& \tilde{\varphi}=\left(\begin{array}{ccc}
1 & 0 & 0 \\
0 & 1 & 0 \\
0 & 0 & 1 \\
0 & 0 & 0
\end{array}\right), \quad \tilde{\psi}=\left(\begin{array}{llll}
0 & 0 & 0 & 1
\end{array}\right) \\
& \begin{array}{ccccccccc}
0 & \rightarrow & \Gamma_{2} & \stackrel{\tilde{\varphi}}{\rightarrow} & \Gamma_{1} \oplus \Gamma_{2} & \stackrel{\dot{\psi}}{\rightarrow} & \Gamma_{1} & \rightarrow & 0 \\
& & \mathcal{\jmath} & & \mathcal{\varphi} & & \| & & \\
\mathscr{E}_{\Gamma_{1}}: & 0 & \rightarrow M(4,0,5) & \stackrel{\varphi}{\rightarrow} & M(7,3,5)^{\prime} & \stackrel{\psi}{\rightarrow} & \Gamma_{1} & \rightarrow & 0
\end{array}
\end{aligned}
$$

where $\tilde{\varphi}=\left(\begin{array}{l}0 \\ 1\end{array}\right), \tilde{\psi}=\left(\begin{array}{ll}1 & 0\end{array}\right)$.

(4) Let $M$ be a direct summand of the middle term of ${ }_{X} \mathscr{E}, X \neq M(7,3,5)^{\prime}$. If $\operatorname{dim} M=(l, 3,5)$ or $\operatorname{dim} M=(l, 6,5)$, then $M$ is indecomposable. (This is immediate

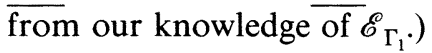

Construct the full subquiver of $\mathscr{A}(\Lambda)$ which is given by $\mathscr{E}_{J^{*}}, \mathscr{E}_{J}, \mathscr{E}_{M(11,6,10)}$, $\mathscr{E}_{M(7,3,5)}, \mathscr{E}_{\Gamma_{2}}, \mathscr{E}_{M(8,6,5)}$ (using (1) and (3)). From this point forth the dimension-type which completes a mesh always belongs to an indecomposable $\Lambda$-lattice (in view of (4)). Continue using this argument until the construction terminates. Q.E.D.

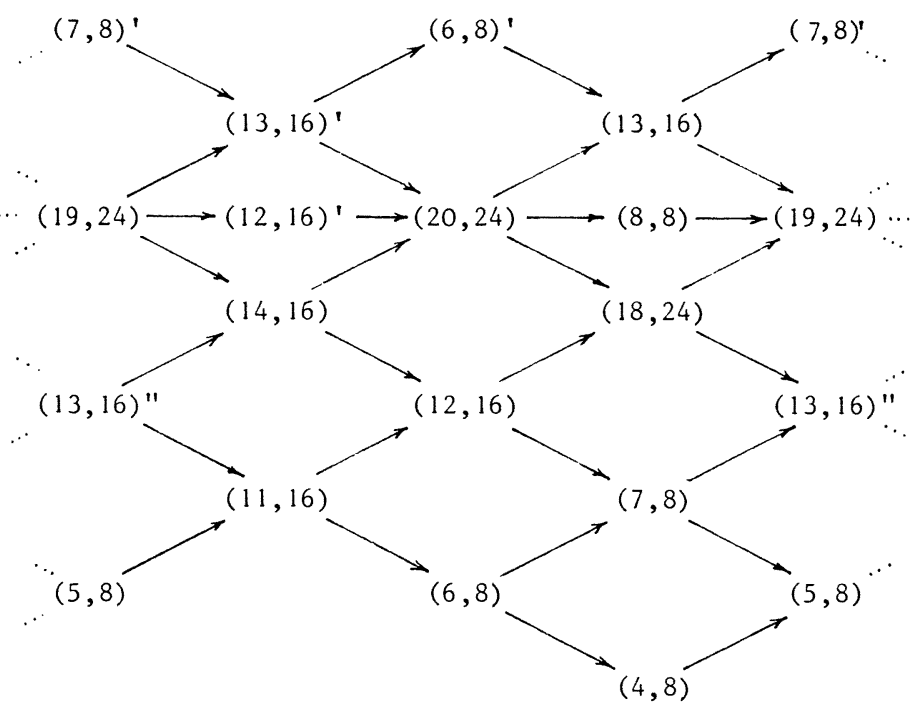

Figure 8 
Proposition 3.7. Let $\Delta=\mathbf{E}_{8}$. Then $\mathscr{A}\left(\Lambda_{\Delta}\right)$ is given by Figure 8 where one has to identify modulo $2 \tilde{\tau}$.

Proof. (1) It is elementary to classify all irreducible $\Lambda$-lattices. Up to isomorphism they are given as follows: $M(4,8)=\Lambda, M(5,8)=J^{*}, M(6,8)=J, M(6,8)^{\prime}=$ $\Lambda+T^{4} \Lambda, M(7,8)=\Lambda+T^{2} \Lambda+T^{4} \Lambda, M(7,8)^{\prime}=\Lambda+T \Lambda, M(8,8)=\Gamma$.

(2) $\tau(J) \cong J^{*}, \tau\left(M(7,8)^{\prime}\right) \cong M(6,8)^{\prime}, \tau\left(M(6,8)^{\prime}\right) \cong M(7,8)^{\prime}$.

(3) The following $\Lambda$-lattices are indecomposable:

$$
\begin{aligned}
& M(13,16)=\left[\left(\Lambda+T^{4} \Lambda\right) \oplus\left(\Lambda+T^{4} \Lambda\right)\right]+\left(T, T^{2}\right) \Lambda \subset \Gamma^{(2)}, \\
& M(13,16)^{\prime}=\left[T \Gamma \oplus\left(\Lambda+T^{7} \Lambda\right)\right]+\left(1, T^{2}\right) \Lambda \subset \Gamma^{(2)}, \\
& M(20,24)=\left[T \Gamma \oplus\left(\Lambda+T^{4} \Lambda\right) \oplus\left(\Lambda+T^{7} \Lambda\right)\right]+(1, T, 0) \Lambda+\left(0, T, T^{2}\right) \Lambda \subset \Gamma^{(3)}, \\
& M(12,16)^{\prime}=\left[\left(\Lambda+T^{4} \Lambda\right) \oplus\left(\Lambda+T^{7} \Lambda\right)\right]+\left(T, T^{2}\right) \Lambda \subset \Gamma^{(2)} .
\end{aligned}
$$

(4) The Auslander-Reiten sequences $\mathscr{E}_{M(7,8)^{\prime}}, \mathscr{E}_{M(13,16)}, \mathscr{E}_{\Gamma}$ are given by the following commutative diagrams:

$$
\begin{aligned}
& \begin{array}{lllllll}
0 & \Gamma & \stackrel{\tilde{\varphi}}{\rightarrow} & \Gamma^{(2)} & \stackrel{\tilde{\psi}}{\rightarrow} & \Gamma & \rightarrow 0 \\
& & & & & \uparrow
\end{array} \\
& \mathscr{E}_{M(7,8)^{\prime}}: \quad 0 \quad \rightarrow M(6,8)^{\prime} \quad \stackrel{\varphi}{\rightarrow} M(13,16) \quad \stackrel{\psi}{\rightarrow} M(7,8)^{\prime} \quad \rightarrow 0
\end{aligned}
$$

where $\tilde{\varphi}=\left(\begin{array}{l}0 \\ 1\end{array}\right), \quad \tilde{\psi}=\left(\begin{array}{ll}1 & 0\end{array}\right)$;

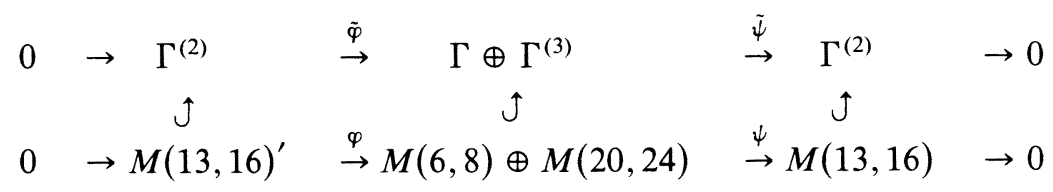

where

$$
\begin{aligned}
& \tilde{\varphi}=\left(\begin{array}{cc}
-T^{2} & 1 \\
\hline 1 & 0 \\
0 & 0 \\
0 & -1
\end{array}\right), \quad \tilde{\psi}=\left(\begin{array}{c|ccc}
0 & 0 & 1 & 0 \\
1 & T^{2} & 0 & 1
\end{array}\right) \\
& 0 \rightarrow \Gamma^{(2)} \quad \stackrel{\tilde{\varphi}}{\rightarrow} \Gamma^{(3)} \quad \stackrel{\tilde{\psi}}{\rightarrow} \Gamma \quad \rightarrow 0
\end{aligned}
$$

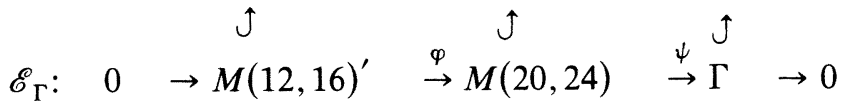

where

$$
\tilde{\varphi}=\left(\begin{array}{ll}
0 & 0 \\
1 & 0 \\
0 & 1
\end{array}\right), \quad \tilde{\psi}=\left(\begin{array}{lll}
1 & 0 & 0
\end{array}\right)
$$


After these preparations, $\mathscr{A}(\Lambda)$ is now easily obtained. We proceed in three steps:

(a) Construct the full subquiver of $\mathscr{A}(\Lambda)$ which is given by the projective mesh and by $\mathscr{E}_{J}$ (using (1) and (2)).

(b) Construct the full subquiver of $\mathscr{A}(\Lambda)$ which is given by $\mathscr{E}_{M(7,8)^{\prime}}, \mathscr{E}_{M(6,8)^{\prime}}$, $\mathscr{E}_{M(13,16)}, \mathscr{E}_{M(13,16)^{\prime}}, \mathscr{E}_{\Gamma}$ (using (4) and (2)).

(c) The full subquivers of $\mathscr{A}(\Lambda)$ constructed in (a) and (b) contain all irreducible $\Lambda$-lattices (in view of (1)). From this it is easy to deduce that henceforth the dimension-type which completes a mesh always belongs to an indecomposable $\Lambda$-lattice. Use this argument repeatedly, until the construction terminates. Q.E.D.

4. Supplementary note. In the meanwhile, J.-L. Verdier has observed that the Auslander-Reiten quivers given in the present paper and the McKay graphs described in [Go/Ve83] coincide, and H. Knörrer has given a conceptual proof for this fact. Moreover, this has motivated H. Knörrer to investigate the AuslanderReiten quiver of the category of Cohen-Macaulay modules over the complete local ring of a simple hypersurface singularity of arbitrary dimension $n$ : He gives a natural explanation for the twisting construction described in the present paper and he proves that for dimensions $n$ and $n+2$ the respective stable Auslander-Reiten quivers coincide.

\section{REFERENCES}

[Ar74] V. I. Arnol'd, Critical points on smooth functions, Proc. Inernat. Congr. Math. Vancouver 1974, vol. 1, pp. 19-39.

[Au75] M. Auslander, Existence theorems for almost split sequences, Ring Theory II (Proc. Second Oklahoma Conf.), Dekker, New York, 1975, pp. 1-44.

[Ba63] H. Bass, On the ubiquity of Gorenstein rings, Math. Z. 82 (1963), 8-28.

[Bo/Ga82] K. Bongartz and P. Gabriel, Covering spaces in representation-theory, Invent. Math. 65 (1982), 331-678.

[Bu79] M. C. R. Butler, The construction of almost split sequences II: Lattices over orders, Bull. London Math. Soc. 11 (1979), 155-160.

[Di83] E. Dieterich, Construction of Auslander-Reiten quivers for a class of group rings, Math. Z. 184 (1983), 43-60.

[Ga80] P. Gabriel, Auslander-Reiten sequences and representation-finite algebras, Proc. Ottawa Conf. Representation Theory (Ottawa 1979), Lecture Notes in Math., vol. 831, Springer, Berlin and New York, 1980, pp. 1-71.

[Go/Ve83] G. Gonzalez-Sprinberg and J.-L. Verdier, Construction géométrique de la correspondence de McKay, Ann. Sci. Ecole Norm. Sup. 16 (1983), 409-449.

[Gr/Kn85] G. M. Greuel and H. Knörrer, Einfache Kurvensingularitäten und torsionsfreie Moduln, Math. Ann. 270 (1985), 417-425.

[Gr/Re78] E. Green and I. Reiner, Integral representation and diagrams, Michigan Math. J. 25 (1978), 53-84.

[Re/Rie85] I. Reiten and C. Riedtmann, Skew group algebras in the representation theory of artin algebras, J. Algebra 92 (1985), 224-282.

[Rie80] C. Riedtmann, Algebren, Darstellungsköcher, Überlagerungen und zurück, Comment. Math. Helv. 55 (1980), 199-224.

[Rie80a] _ Representation-finite selfinjective algebras of class $A_{n}$, Proc. Ottawa Conf. Representation Theory (Ottawa 1979), Lecture Notes in Math., vol. 832, Springer, Berlin and New York, 1980, pp. 449-520.

[Rin80] C. M. Ringel, Tame algebras, Proc. Ottawa Conf. Representation Theory (Ottawa 1979), Lecture Notes in Math., vol. 831, Springer, Berlin and New York, 1980, pp. 137-287.

[Rin/Ro79] C. M. Ringel and K. W. Roggenkamp, Diagramatic methods in the representation theory of orders, J. Algebra 60 (1979), 11-42. 
[Ro77] K. W. Roggenkamp, The construction of almost split sequences for integral group rings and orders, Comm. Algebra 5 (1977), 1363-1373.

[Ro81] _ The lattice type of orders II: Auslander-Reiten quivers, Proc. Conf. Integral Representations and Applications (Oberwolfach 1980), Lecture Notes in Math., vol. 882, Springer, Berlin and New York, 1981, pp. 430-478.

[Wi82] A. Wiedemann, The Auslander-Reiten graph of integral blocks with cyclic defect two and their integral representations, Math. Z. 179 (1982), 407-429.

[Wi84] __ Classification of the Auslander-Reiten quivers of local Gorenstein orders and a characterization of the Kleinian singularities (preprint).

UNiversität Bielefeld, FakUltät FƯr MAthematik, UniversitätsStrasSE, 4800 Bielefeld 1, FEDERAL RePUBlic OF GERMANY

Universität Stuttgart, Mathematisches Institut, Pfaffenwaldring 57, 7000 Stuttgart 80 , Federal Republic of Germany (Current aaddress of Alfred Wiedemann)

Current address (Ernst Dieterich): Department of Mathematics, Brandeis University, Waltham, Massachusetts 02254 